INTERNATIONAL JOURNAL FOR NUMERICAL METHODS IN FLUIDS

Int. J. Numer. Meth. Fluids (2008)

Published online in Wiley InterScience (www.interscience.wiley.com). DOI: 10.1002/fld.1824

\title{
Direct optimal growth analysis for timesteppers
}

\author{
D. Barkley ${ }^{1,2}$, H. M. Blackburn ${ }^{3}$ and S. J. Sherwin ${ }^{4, *, \dagger}$ \\ ${ }^{1}$ Mathematics Institute, University of Warwick, Coventry CV4 7AL, U.K. \\ ${ }^{2}$ PMMH UMR 7636 de l'ESPCI, 10 rue Vauquelin, 75231 Paris Cedex 5, France \\ ${ }^{3}$ Department of Mechanical and Aerospace Engineering, Monash University, Vic. 3800, Australia \\ ${ }^{4}$ Department of Aeronautics, Imperial College London, London SW7 2AZ, U.K.
}

\begin{abstract}
SUMMARY
Methods are described for transient growth analysis of flows with arbitrary geometric complexity, where in particular the flow is not required to vary slowly in the streamwise direction. Emphasis is on capturing the global effects arising from localized convective stability in streamwise-varying flows. The methods employ the 'timestepper's approach' in which a nonlinear Navier-Stokes code is modified to provide evolution operators for both the forward and adjoint linearized equations. First, the underlying mathematical treatment in primitive flow variables is presented. Then, details are given for the inner level code modifications and outer level eigenvalue and SVD algorithms in the timestepper's approach. Finally, some examples are shown and guidance provided on practical aspects of this type of large-scale stability analysis. Copyright (C) 2008 John Wiley \& Sons, Ltd.
\end{abstract}

Received 1 October 2007; Revised 17 March 2008; Accepted 17 March 2008

KEY WORDS: large-scale stability analysis; transient growth; adjoint; iterative eigenvalue problem; linearized Navier-Stokes; SVD

\section{INTRODUCTION}

Methods developed for direct linear stability analysis of the Navier-Stokes equations in general geometries have been previously described in detail [1] and extensively applied [2-8]. Only recently, however, have large-scale techniques been extended to the transient growth problem [9-12]. Here, we present methods for direct optimal growth computation for the linearized NavierStokes equations in general geometries. Although our implementations have employed spectral/ $h p$ element formulations [13], the methods we present are generally applicable for any mesh-based

\footnotetext{
*Correspondence to: S. J. Sherwin, Department of Aeronautics, Imperial College London, South Kensington Campus, London SW7 2AZ, U.K.

${ }^{\dagger}$ E-mail: s.sherwin@imperial.ac.uk

Contract/grant sponsor: Senior Fellowship of the City of Paris

Contract/grant sponsor: U.K. EPSRC; contract/grant number: EP/E006493/1
}

Copyright (C) 2008 John Wiley \& Sons, Ltd. 

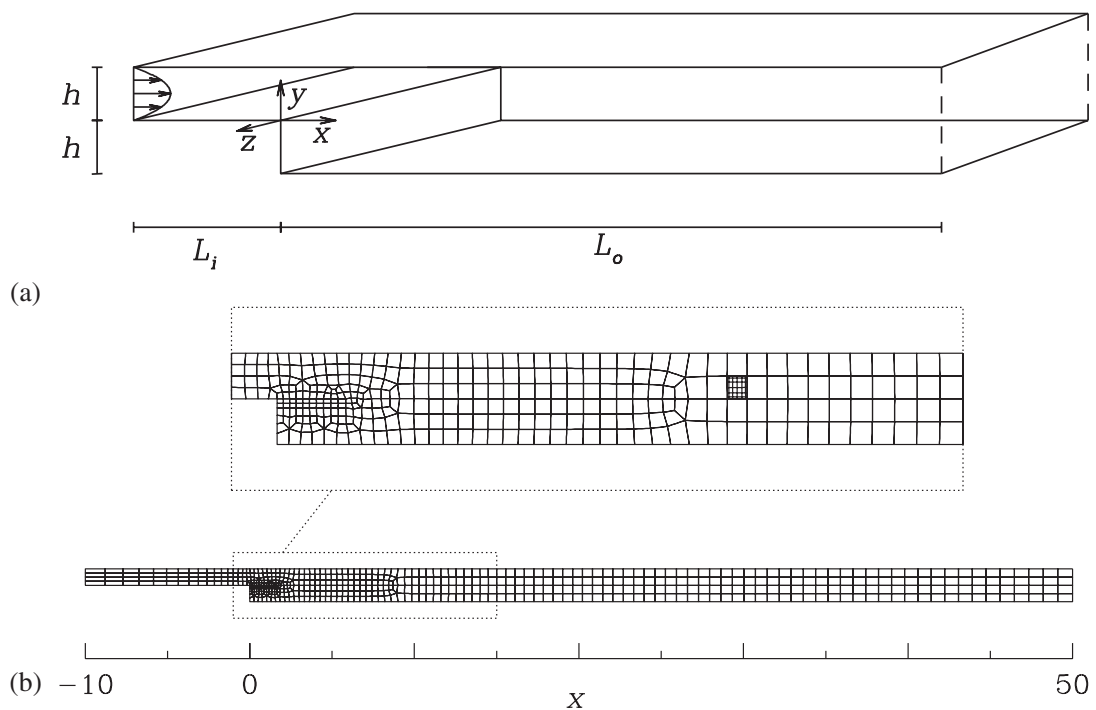

Figure 1. An example flow domain $\Omega$ and its discretization into spectral elements. (a) The backwardfacing step geometry can be considered as either three dimensional or, by restriction to only $(x, y)$ coordinates, two dimensional. (b) This shows a two-dimensional spectral element mesh for such a geometry, with inset detail of the region near the step and collocation grid points for a polynomial order $N=6$ within an example element.

discretization. Similar to [1], we will adopt a 'bifurcation analysis for timesteppers' approach that allows these analyses to be undertaken after a reasonably straightforward modification of a time-stepping Navier-Stokes algorithm. This approach has the benefit not only of leveraging an existing code base to solve transient growth problems but also of providing a unified code for a variety of linear and nonlinear computations.

Consider the general situation of incompressible flow in a domain $\Omega$ in two or three dimension. The fluid motion is governed by the incompressible Navier-Stokes equations:

$$
\begin{gathered}
\partial_{t} \mathbf{u}=-(\mathbf{u} \cdot \nabla) \mathbf{u}-\nabla p+R e^{-1} \nabla^{2} \mathbf{u} \quad \text { in } \Omega \\
\nabla \cdot \mathbf{u}=0 \quad \text { in } \Omega
\end{gathered}
$$

where $\mathbf{u}(\mathbf{x}, t)=(u, v, w)(x, y, z, t)$ is the velocity field and $p(\mathbf{x}, t)$ is the (modified, or kinematic) pressure field. Additionally, it is necessary to specify appropriate boundary conditions. These may correspond to a closed flow, such as flow in a cavity, or to an open flow such as flow past a bluff body or flow through a channel. Figure 1 illustrates one example, which we shall primarily use in this paper.

Our starting point is a base flow $\mathbf{U}$ which is a steady or time-varying solution to (1) with associated pressure $P$. We are specifically interested in situations in which geometry dictates a base flow that is inaccessible analytically, and particularly we emphasize open systems whose base flows vary substantially in the streamwise direction. Such base flows are typically computed by direct numerical simulation of (1) or by some fixed-point method such as Newton's method. These methods are discussed extensively elsewhere; here, we shall just assume that $\mathbf{U}$ is accurately 
computable in $\Omega$ by some technique. Where the base flow is time dependent, we will be primarily concerned with cases that are time-periodic, although more general situations are possible. In our formulation, it will not generally be necessary to distinguish between steady and unsteady cases nor between two- and three-dimensional base flows.

What follows concerns the evolution of infinitesimal perturbations $\mathbf{u}^{\prime}$ to the base flows. The equations governing these perturbations are found by inserting $\mathbf{u}=\mathbf{U}+\varepsilon \mathbf{u}^{\prime}$ and $p=P+\varepsilon p^{\prime}$, where $p^{\prime}$ is the pressure perturbation, into (1) and keeping the lowest order (linear) terms in $\varepsilon$. The resulting linearized Navier-Stokes equations are

$$
\begin{gathered}
\partial_{t} \mathbf{u}^{\prime}=-(\mathbf{U} \cdot \nabla) \mathbf{u}^{\prime}-\left(\mathbf{u}^{\prime} \cdot \nabla\right) \mathbf{U}-\nabla p^{\prime}+R e^{-1} \nabla^{2} \mathbf{u}^{\prime} \quad \text { in } \Omega \\
\nabla \cdot \mathbf{u}^{\prime}=0 \quad \text { in } \Omega
\end{gathered}
$$

These equations are solved subject to appropriate initial and boundary conditions as discussed below.

There are two important questions concerning solutions to (2). The first question is: do there exist any solutions that grow without bound? If so, then the base flow $\mathbf{U}$ is linearly unstable. The second question is: given a base flow which is linearly stable, do there exist bounded solutions of (2) that exhibit large transient growth before they inevitably decay? In such cases, the flow is linearly stable but might have local regions of convective instability, or it might be susceptible to nonlinear instability, or both. In answering either question, we wish to find the perturbation that generates optimal growth, in the long term for the first case or for a finite term in the second case.

For example, Figure 2 illustrates the temporal evolution of an optimal two-dimensional transient disturbance to steady two-dimensional flow over a backward-facing step. At the inflow Reynolds number $R e=U_{\mathrm{c}} h / v=500$, the flow is asymptotically stable. However, the flow has localized convective instability and hence exhibits large linear transient growth of suitable disturbances-an

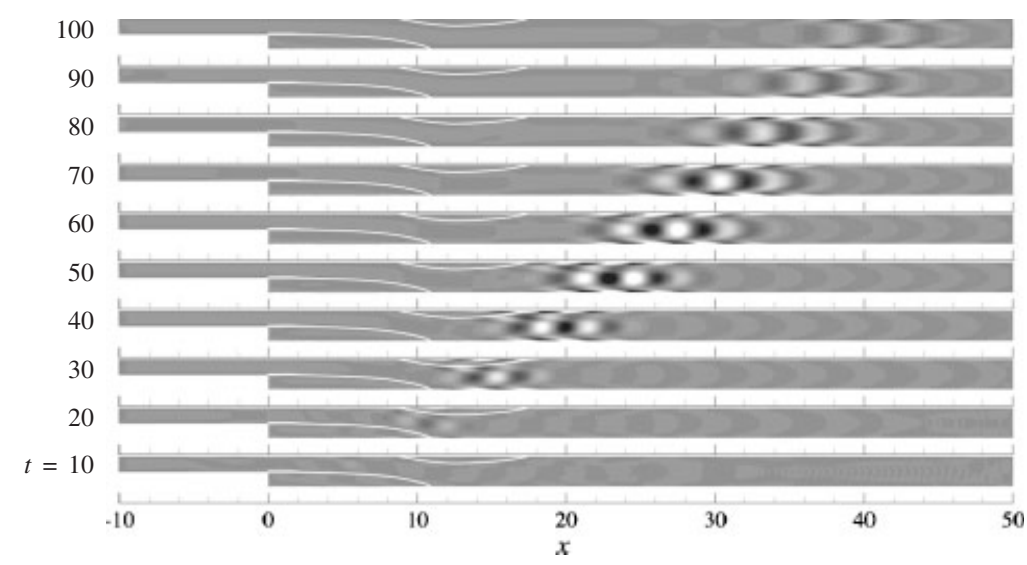

Figure 2. Sequence of linear perturbation vorticity contours developed from the two-dimensional global optimum disturbance initial condition for $R e=500$ in flow over a backward-facing step. Maximum energy growth occurs for $t=\tau=58.0$, when the centroid of perturbation energy lies at $x=26.4$. The separation streamlines of the base flow are also shown. 


\section{BARKLEY, H. M. BLACKBURN AND S. J. SHERWIN}

association first made by Cossu and Chomaz [14]. The focus of the present work is on methods for determining the optimal flow disturbance in such general (non-parallel) flows, with emphasis on transient cases arising from localized regions of instability.

\subsection{Linear stability}

Equations (2) define a linear evolution operator $\mathscr{A}$, which evolves perturbations forward in time:

$$
\mathbf{u}^{\prime}(t)=\mathscr{A}(t) \mathbf{u}^{\prime}(0)
$$

It is through this forward evolution operator that we gain access to linear stability of the base flow. If the base flow $\mathbf{U}$ is steady, then perturbation equations (2) are autonomous and eigenmode solutions will be of the form $\mathbf{u}^{\prime}(\mathbf{x}, t)=\exp \left(\lambda_{j} t\right) \tilde{\mathbf{u}}_{j}(\mathbf{x})+$ c.c., where in general $\lambda_{j}$ and $\tilde{\mathbf{u}}_{j}$ are complex. If the base flow is periodic with period $T$, then (2) becomes a Floquet problem and the Floquet solutions will be of the form $\mathbf{u}^{\prime}(x, t)=\exp \left(\lambda_{j} t\right) \tilde{\mathbf{u}}_{j}(\mathbf{x}, t)+$ c.c., where $\tilde{\mathbf{u}}_{j}(\mathbf{x}, t+T)=\tilde{\mathbf{u}}_{j}(\mathbf{x}, t)$. In either case, by setting the time $t$ in (3) to a value $T$, we obtain an eigenvalue problem in terms of the operator $\mathscr{A}(T)$ :

$$
\mathscr{A}(T) \tilde{\mathbf{u}}_{j}=\mu_{j} \tilde{\mathbf{u}}_{j}, \quad \mu_{j} \equiv \exp \left(\lambda_{j} T\right)
$$

If the base flow is $T$-periodic, then $\mu_{j}$ is called a Floquet multiplier and the eigenmodes of $\mathscr{A}$ are the Floquet modes evaluated at a specific temporal phase. If the base flow is steady, then $T$ is an arbitrary positive value (chosen on practical grounds, i.e. to allow the perturbation to evolve for a reasonable time) and one would normally be interested in the values $\lambda_{j}$ rather than $\mu_{j}$. In the latter case, the eigenmodes of $\mathscr{A}$ are the same as those of the linearized Navier-Stokes equations.

Classical linear stability of the base flow is then determined from the dominant eigenvalues of $\mathscr{A}(T)$, i.e. $\mu_{i}$ of the largest modulus. If there are any eigenvalues $\mu_{j}$ with $\left|\mu_{j}\right|>1$, then there exist exponentially growing solutions of (2) and, hence, the base flow is linearly unstable. Conversely, if every eigenvalue satisfies $\left|\mu_{j}\right|<1$, then every solution of (2) eventually decays to zero and the flow is linearly stable. Generally, $\left|\mu_{j}\right|=1$ would signal a bifurcation point.

\subsection{Transient growth and the SVD problem}

We now consider the transient evolution of perturbations. More specifically, we are interested in situations in which the base flow is linearly stable, but perturbations exhibit substantial transient response due to regions of localized convective instability. This situation is common in many open flows, particularly those for which geometrical complexity dictates a rapid variation in the underlying base flow. Owing to the non-orthogonality of the eigenmodes of $\mathscr{A}$, which stems from the asymmetry of the convective terms in the Navier-Stokes equations, the dynamics of interest may not be of the form $\exp (\lambda t) \tilde{\mathbf{u}}(\mathbf{x})$, i.e. an exponential function of time multiplying a fixed modal shape; therefore, eigenvalue problem (4) is simply not directly relevant $[15,16]$. Instead, the way to quantify such dynamics is through a transient growth analysis or, equivalently, through a computation of the singular values and singular vectors of $\mathscr{A}$.

First, it is necessary to choose a norm to quantify the size of a perturbation, A physically meaningful choice, which is also the most commonly used, is based on the total kinetic energy of a perturbation field over the flow domain $\Omega$. The energy norm also has the mathematical advantage 


\section{DIRECT OPTIMAL GROWTH}

of being the norm which is associated with the standard $L_{2}$ inner product:

$$
(\mathbf{u}, \mathbf{v}) \equiv \int_{\Omega} \mathbf{u} \cdot \mathbf{v} \mathrm{d} v
$$

(Note we will only take the inner product of real-valued fields; therefore, complex conjugation does not appear.) Transient growth is measured as the perturbation energy at time $\tau$ normalized to its initial energy. Since the perturbation equations are linear, we can, without loss of generality, simplify the algebra and consider normalized initial perturbations, e.g.

$$
\frac{E(\tau)}{E(0)}=\left\|\mathbf{u}^{\prime}(\tau)\right\|^{2}=\left(\mathbf{u}^{\prime}(\tau), \mathbf{u}^{\prime}(\tau)\right), \quad\left\|\mathbf{u}^{\prime}(0)\right\|=1
$$

Using the evolution operator $\mathscr{A}(\tau)$ this can be expressed as

$$
\frac{E(\tau)}{E(0)}=\left(\mathscr{A}(\tau) \mathbf{u}^{\prime}(0), \mathscr{A}(\tau) \mathbf{u}^{\prime}(0)\right)=\left(\mathbf{u}^{\prime}(0), \mathscr{A}^{*}(\tau) \mathscr{A}(\tau) \mathbf{u}^{\prime}(0)\right)
$$

where in the last equality $\mathscr{A}^{*}(\tau)$ is the adjoint evolution operator to $\mathscr{A}(\tau)$ in the $L_{2}$ inner product. This adjoint operator is discussed in detail in Section 2.

As with the linear stability problem, here too we are interested in the 'worst case scenario.' That is, for the stability problem, we are almost exclusively interested in the dominant and near dominant eigenvalues $\mu_{j}$ of the largest magnitude since these dictate linear stability. For the transient growth problem, the pertinent question is: what are the perturbations that lead to maximal or near maximal growth? From (5), the eigenfunction corresponding to the dominant eigenvalues of $\mathscr{A}^{*}(\tau) \mathscr{A}(\tau)$ will dictate the largest possible growth. Letting $\lambda_{j}$ and $\mathbf{v}_{j}$ denote eigenvalues and normalized eigenfunctions, we have

$$
\mathscr{A}^{*}(\tau) \mathscr{A}(\tau) \mathbf{v}_{j}=\lambda_{j} \mathbf{v}_{j}, \quad\left\|\mathbf{v}_{j}\right\|=1
$$

Typically, $G(\tau)$ denotes the maximum growth obtainable at time $\tau$, in which case

$$
G(\tau) \equiv \max _{\left\|\mathbf{u}^{\prime}(0)\right\|=1} \frac{E(\tau)}{E(0)}=\max _{j} \lambda_{j}
$$

Although the maximum eigenvalue of $\mathscr{A}^{*}(\tau) \mathscr{A}(\tau)$ is the most significant, the first few sub-dominant eigenvalues may also be of interest.

Finding the dominant eigenvalues of $\mathscr{A}^{*}(\tau) \mathscr{A}(\tau)$ is equivalent to finding the largest singular values of $\mathscr{A}(\tau)$, and this has a direct physical interpretation. The eigenfunction $\mathbf{v}_{j}$ in (6) provides an initial perturbation $\mathbf{u}^{\prime}(0)$, which generates a growth $\lambda_{j}$ over time $\tau$. However, this is only half the story as one is also interested in $\mathbf{u}^{\prime}(\tau)$, the perturbation at time $\tau$. Defining $\mathbf{u}_{j}$ to be the normalized perturbation at time $\tau$ evolved from initial condition $\mathbf{u}^{\prime}(0)=\mathbf{v}_{j}$, we have

$$
\mathscr{A}(\tau) \mathbf{v}_{j}=\sigma_{j} \mathbf{u}_{j}, \quad\left\|\mathbf{u}_{j}\right\|=1
$$

where $\sigma_{j}=\left\|\mathbf{u}^{\prime}(\tau)\right\|$. This is nothing other than the singular value decomposition of $\mathscr{A}(\tau)$. A few readily verifiable facts are as follows: the right singular vectors $\mathbf{v}_{j}$ are real and orthonormal in $L_{2}$ (since $\mathscr{A}^{*}(\tau) \mathscr{A}(\tau)$ is self-adjoint), likewise the left singular vectors $\mathbf{u}_{j}$ are real and orthonormal, $\lambda_{j}$ and $\sigma_{j}$ are real and non-negative, and $\sigma_{j}=\lambda_{j}^{1 / 2}$ are the singular values of $\mathscr{A}(\tau)$. 


\subsection{Discussion}

In the 'bifurcation for timesteppers' approach previously outlined in [1], one first modifies a code so as to approximate the action of the evolution operator $\mathscr{A}(\tau)$ defined in $(3)$. Then, one uses an eigenvalue solver for the eigenvalue problem (4) to obtain the dominant eigenvalues and eigenmodes. Our primary focus in the present paper is on the finite-time optimal growth problem (6). In fact, this problem is very similar to the classical linear stability computation apart from the fact that we are interested in the maximum eigenvalue of $\mathscr{A}^{*}(\tau) \mathscr{A}(\tau)$ rather than of $\mathscr{A}(\tau)$. By simple extensions to the original timestepper approach, one can also solve the optimal growth problem, and the main purpose here is to describe the theoretical and practical aspects of these extensions.

\section{MATHEMATICAL FUNDAMENTALS}

The goal of this section is to set out clearly the relationship between the linearized Navier-Stokes equations (2) and its associated evolution operator $\mathscr{A}(\tau)$, and the adjoint linearized Navier-Stokes equations and its evolution operator $\mathscr{A}^{*}(\tau)$. In so doing, we consider issues associated with boundary conditions of the two systems. Our intention in the following is to be as complete as possible without going into too many formal mathematical details. This material is not original, but is provided here to have available a comprehensive treatment in a primitive-variables formulation. We will refer to the linearized Navier-Stokes equations together with appropriate initial and boundary conditions as the forward system and the adjoint linearized Navier-Stokes equations, to be introduced, together with appropriate adjoint initial and boundary conditions as the adjoint system.

\subsection{Variables and inner products}

For considering the equations expressed in primitive variables, it is convenient to define the quantities

$$
\mathbf{q}\left(\begin{array}{l}
\mathbf{u}^{\prime} \\
p^{\prime}
\end{array}\right), \quad \mathbf{q}^{*}=\left(\begin{array}{c}
\mathbf{u}^{*} \\
p^{*}
\end{array}\right)
$$

where $\mathbf{u}^{\prime}$ and $p^{\prime}$ are the perturbation velocity field and pressure, and $\mathbf{u}^{*}$ and $p^{*}$ denote the adjoint velocity and pressure fields. All fields are real valued and the star is only notational; it does not denote complex conjugate.

We let $\Gamma=\Omega \times(0, \tau)$ be the space-time domain on which the equations are posed, where $\Omega$ is the spatial domain and $\tau$ is some final time, arbitrary other than that $\tau>0$. Associated with domain $\Gamma$ is the inner product:

$$
\left\langle\mathbf{q}, \mathbf{q}^{*}\right\rangle=\int_{0}^{\tau} \int_{\Omega} \mathbf{q} \cdot \mathbf{q}^{*} \mathrm{~d} v \mathrm{~d} t
$$

By contrast, the linear evolution operator $\mathscr{A}(\tau)$ and adjoint evolution operator $\mathscr{A}^{*}(\tau)$ act on velocity fields $\mathbf{u}^{\prime}$ and $\mathbf{u}^{*}$, respectively. For these, the relevant domain is just the spatial domain $\Omega$ and the 
inner product is that already given in Section 1.2:

$$
\left(\mathbf{u}^{\prime}, \mathbf{u}^{*}\right)=\int_{\Omega} \mathbf{u}^{\prime} \cdot \mathbf{u}^{*} \mathrm{~d} v
$$

\subsection{Operators and PDEs}

By defining certain operators, we will be able to write concisely and manipulate simply the linearized Navier-Stokes and adjoint equations. Let $\mathscr{H}$ be defined as

$$
\mathscr{H}=\left[\begin{array}{c|c}
-\partial_{t}-\mathrm{DN}+R e^{-1} \nabla^{2} & -\nabla \\
\hline \nabla \cdot & 0
\end{array}\right]
$$

with the linearized advection operator DN defined as

$$
\mathrm{DNu}^{\prime}=(\mathbf{U} \cdot \nabla) \mathbf{u}^{\prime}+\left(\mathbf{u}^{\prime} \cdot \nabla\right) \mathbf{U}=(\mathbf{U} \cdot \nabla) \mathbf{u}^{\prime}+(\nabla \mathbf{U}) \cdot \mathbf{u}^{\prime}
$$

Then the linearized Navier-Stokes equations (2) can be expressed compactly as

$$
\mathscr{H} \mathbf{q}=0
$$

The adjoint of $\mathscr{H}$ in inner product (9) is

$$
\mathscr{H}^{*}=\left[\begin{array}{c|c}
\partial_{t}-\mathrm{DN}^{*}+R e^{-1} \nabla^{2} & -\nabla \\
\hline \nabla \cdot & 0
\end{array}\right]
$$

where $\mathrm{DN}^{*}$, the adjoint linearized advection operator, is

$$
\mathrm{DN}^{*} \mathbf{u}^{*}=-(\mathbf{U} \cdot \nabla) \mathbf{u}^{*}+(\nabla \mathbf{U})^{\mathrm{T}} \cdot \mathbf{u}^{*}
$$

The justification follows from the requirement that for $\mathscr{H}^{*}$ to be the adjoint of $\mathscr{H}$, then by definition

$$
\left\langle\mathscr{H} \hat{\mathbf{q}}, \hat{\mathbf{q}}^{*}\right\rangle-\left\langle\hat{\mathbf{q}}, \mathscr{H}^{*} \hat{\mathbf{q}}^{*}\right\rangle=0
$$

for any fields $\hat{\mathbf{q}}, \hat{\mathbf{q}}^{*}$ with compact support (so that all values and derivatives are zero on all the boundaries) within the domain $\Gamma$. Integration by parts gives

$$
\begin{aligned}
\left\langle\mathscr{H} \hat{\mathbf{q}}, \hat{\mathbf{q}}^{*}\right\rangle-\left\langle\hat{\mathbf{q}}, \mathscr{H}^{*} \hat{\mathbf{q}}^{*}\right\rangle= & \int_{0}^{\tau} \int_{\Omega}-\partial_{t}\left(\mathbf{u} \cdot \mathbf{u}^{*}\right) \mathrm{d} v \mathrm{~d} t+\int_{0}^{\tau} \int_{\Omega} \nabla \cdot\left\{-\mathbf{U}\left(\mathbf{u} \cdot \mathbf{u}^{*}\right)\right. \\
& \left.+\mathbf{u} p^{*}-\mathbf{u}^{*} p+\operatorname{Re}^{-1}\left((\nabla \mathbf{u})^{\mathrm{T}} \cdot \mathbf{u}^{*}-\left(\nabla \mathbf{u}^{*}\right)^{\mathrm{T}} \cdot \mathbf{u}\right)\right\} \mathrm{d} v \mathrm{~d} t
\end{aligned}
$$

Employing the divergence theorem, this can be expressed using only boundary terms:

$$
\begin{aligned}
\left\langle\mathscr{H} \hat{\mathbf{q}}, \hat{\mathbf{q}}^{*}\right\rangle-\left\langle\hat{\mathbf{q}}, \mathscr{H}^{*} \hat{\mathbf{q}}^{*}\right\rangle= & -\int_{\Omega}\left[\mathbf{u} \cdot \mathbf{u}^{*}\right]_{0}^{\tau} \mathrm{d} v+\int_{0}^{\tau} \oint_{\partial \Omega} \hat{\mathbf{n}} \cdot\left\{-\mathbf{U}\left(\mathbf{u} \cdot \mathbf{u}^{*}\right)\right. \\
& \left.+\mathbf{u} p^{*}-\mathbf{u}^{*} p+R e^{-1}\left((\nabla \mathbf{u})^{\mathrm{T}} \cdot \mathbf{u}^{*}-\left(\nabla \mathbf{u}^{*}\right)^{\mathrm{T}} \cdot \mathbf{u}\right)\right\} \mathrm{d} S \mathrm{~d} t
\end{aligned}
$$

where $\hat{\mathbf{n}}$ is a unit outward normal on the spatial boundary of the domain, $\partial \Omega$. For $\hat{\mathbf{q}}$, $\hat{\mathbf{q}}^{*}$ with compact support within $\Gamma$, all boundary terms will vanish and hence (16) is satisfied. 
The adjoint equations corresponding to the linearized Navier-Stokes equations (2) can hence be expressed compactly as

$$
\mathscr{H}^{*} \mathbf{q}^{*}=0
$$

or in full as

$$
\begin{gathered}
-\partial_{t} \mathbf{u}^{*}=-(\mathbf{U} \cdot \nabla) \mathbf{u}^{*}+(\nabla \mathbf{U})^{\mathrm{T}} \cdot \mathbf{u}^{*}-\nabla p^{*}+R e^{-1} \nabla^{2} \mathbf{u}^{*} \\
\nabla \cdot \mathbf{u}^{*}=0
\end{gathered}
$$

The argument above establishes operator $\mathscr{H}^{*}$ as the adjoint of the linearized Navier-Stokes operator $\mathscr{H}$ but does not assist in deriving appropriate sets of initial and boundary conditions.

\subsection{Boundary and initial conditions}

We can now address the initial and boundary conditions for the forward and adjoint systems. We use 'boundary data' to refer to combined initial and boundary conditions, i.e. the data specified on the boundary of the space-time domain. We assume that appropriate boundary data are specified for the forward system and derive the corresponding boundary data for the adjoint problem, which must be compatible with norm (9).

Adjoint boundary data are derived as follows. First, consider fields $\hat{\mathbf{q}}$ that satisfy homogeneous boundary data for the forward system. The homogeneous adjoint boundary data are defined as the minimal homogeneous conditions on the adjoint fields $\hat{\mathbf{q}}^{*}$ such that necessarily

$$
\left\langle\mathscr{H} \hat{\mathbf{q}}, \hat{\mathbf{q}}^{*}\right\rangle-\left\langle\hat{\mathbf{q}}, \mathscr{H}^{*} \hat{\mathbf{q}}^{*}\right\rangle=0
$$

This requirement dictates the type of boundary data for the adjoint system, e.g. whether the conditions are Dirichlet or Neumann type and on which boundaries. This condition does not entirely dictate whether in the end one considers homogeneous or inhomogeneous boundary values. We return to this issue momentarily.

Using integration by parts and the divergence theorem exactly as in Section 2.2,

$$
\begin{aligned}
\left\langle\mathscr{H} \hat{\mathbf{q}}, \hat{\mathbf{q}}^{*}\right\rangle-\left\langle\hat{\mathbf{q}}, \mathscr{H}^{*} \hat{\mathbf{q}}^{*}\right\rangle= & -\int_{\Omega}\left[\mathbf{u} \cdot \mathbf{u}^{*}\right]_{0}^{\tau} \mathrm{d} v+\int_{0}^{\tau} \oint_{\partial \Omega} \hat{\mathbf{n}} \cdot\left\{-\mathbf{U}\left(\mathbf{u} \cdot \mathbf{u}^{*}\right)\right. \\
& \left.+\mathbf{u} p^{*}-\mathbf{u}^{*} p+R e^{-1}\left((\nabla \mathbf{u})^{\mathrm{T}} \cdot \mathbf{u}^{*}-\left(\nabla \mathbf{u}^{*}\right)^{\mathrm{T}} \cdot \mathbf{u}\right)\right\} \mathrm{d} S \mathrm{~d} t
\end{aligned}
$$

For homogeneous Dirichlet boundary data on the forward system,

$$
\mathbf{u}(\mathbf{x}, 0)=0, \quad \mathbf{u}(\partial \Omega, t)=0
$$

the integrals on the right-hand side of Equation (20) become

$$
-\int_{\Omega} \mathbf{u}(\tau) \cdot \mathbf{u}^{*}(\tau) \mathrm{d} v+\int_{0}^{\tau} \oint_{\partial \Omega} \hat{\mathbf{n}} \cdot\left\{-\mathbf{u}^{*} p+\operatorname{Re}^{-1}(\nabla \mathbf{u})^{\mathrm{T}} \cdot \mathbf{u}^{*}\right\} \mathrm{d} S \mathrm{~d} t
$$

The homogeneous adjoint boundary data required to force both integrals to vanish identically are

$$
\mathbf{u}^{*}(\mathbf{x}, \tau)=0, \quad \mathbf{u}^{*}(\partial \Omega, t)=0
$$

Hence, the type of boundary conditions on the adjoint and forward systems are the same: Dirichlet. (See Appendix A for adjoint spatial boundary conditions corresponding to a Neumann outflow 


\section{DIRECT OPTIMAL GROWTH}

condition.) The initial conditions for the adjoint problem are, however, imposed at time $\tau$ and not time zero. This is consistent with the change of sign in front of the time derivative in (18a) relative to $(2 a)$.

As already noted, the definition of the adjoint boundary data dictates only the form of the boundary data. In practice, one would never consider strictly homogeneous boundary data on the forward and adjoint systems as in (21) and (23) because these would only result in the trivial solution. One is, in fact, interested in the case of homogeneous spatial boundary conditions, but inhomogeneous initial conditions i.e. $\mathbf{u}(t=0)=\mathbf{u}_{0} \neq 0$ and $\mathbf{u}^{*}(t=\tau)=\mathbf{u}_{\tau}^{*} \neq 0$. For this case, the forward and adjoint systems, including boundary and initial conditions, which necessarily sets the second integral in Equation (22) to zero, are

$$
\begin{array}{cc}
\text { Forward system } & \text { Adjoint system } \\
\mathscr{H} \mathbf{q}=0 \quad(\mathbf{x}, t) \in \Gamma & \mathscr{H}^{*} \mathbf{q}^{*}=0 \quad(\mathbf{x}, t) \in \Gamma \\
\mathbf{u}(t=0)=\mathbf{u}_{0} & \mathbf{u}^{*}(t=\tau)=\mathbf{u}_{\tau}^{*} \\
\mathbf{u}(\partial \Omega)=0 & \mathbf{u}^{*}(\partial \Omega)=0
\end{array}
$$

\subsection{Evolution operators $\mathscr{A}(\tau)$ and $\mathscr{A}^{*}(\tau)$}

Solutions to the forward and adjoint systems define two evolution operators:

$$
\mathbf{u}(t+s)=\mathscr{A}(s) \mathbf{u}(t), \quad \mathbf{u}^{*}(t-s)=\mathscr{A}^{*}(s) \mathbf{u}^{*}(t)
$$

Because homogeneous boundary conditions are imposed on the forward and adjoint systems, operator $\mathscr{A}^{*}$ is in fact the adjoint of $\mathscr{A}$ under the purely spatial inner product (10). To show this, note that for $\mathbf{q}$ and $\mathbf{q}^{*}$ solving the forward and adjoint systems (24) we have

$$
\left\langle\mathscr{H} \mathbf{q}, \mathbf{q}^{*}\right\rangle-\left\langle\mathbf{q}, \mathscr{H}^{*} \mathbf{q}^{*}\right\rangle=0
$$

Despite the similarity to (16) and (19), this equation holds for a fundamentally different reason, and this is a key point. In (25) each term vanishes identically because we consider solutions $\mathbf{q}$ and $\mathbf{q}^{*}$ to differential equations constructed such that $\mathscr{H} \mathbf{q} \equiv 0$ and $\mathscr{H}^{*} \mathbf{q}^{*} \equiv 0$. In (16) and (19) it was not assumed that $\hat{\mathbf{q}}$ and $\hat{\mathbf{q}}^{*}$ satisfy the differential equations; the space-time boundary terms alone were chosen to force those relations to hold.

Once again we use integration by parts and the divergence theorem to evaluate (25). Because we consider homogeneous spatial boundary conditions, but inhomogeneous initial conditions on both systems, the only terms to survive are those involving time derivatives. Hence,

$$
\int_{\Omega}\left[\mathbf{u}^{\prime} \cdot \mathbf{u}^{*}\right]_{0}^{\tau} \mathrm{d} v=0
$$

Expressing this in terms of inner product (10), we see that (25) implies the following constraint between solutions to the forward and adjoint systems:

$$
\left(\mathbf{u}^{\prime}(\tau), \mathbf{u}^{*}(\tau)\right)=\left(\mathbf{u}^{\prime}(0), \mathbf{u}^{*}(0)\right)
$$

It follows from this that $\mathscr{A}^{*}(\tau)$ is the adjoint of $\mathscr{A}(\tau)$ under inner product $(10)$, since

$$
\left(\mathscr{A}(\tau) \mathbf{u}^{\prime}(0), \mathbf{u}^{*}(\tau)\right)=\left(\mathbf{u}^{\prime}(\tau), \mathbf{u}^{*}(\tau)\right)=\left(\mathbf{u}^{\prime}(0), \mathbf{u}^{*}(0)\right)=\left(\mathbf{u}^{\prime}(0), \mathscr{A}^{*}(\tau) \mathbf{u}^{*}(\tau)\right)
$$


Constraint (26), for the particular case when $\mathbf{u}^{*}(\tau)=\mathscr{A}(\tau) \mathbf{u}^{\prime}(0)$, is used in establishing the relationship between maximum kinetic energy growth and the eigensystem of $\mathscr{A}^{*}(\tau) \mathscr{A}(\tau)$ in Equations (5)-(7).

We note that the action of operator $\mathscr{A}^{*}(\tau) \mathscr{A}(\tau)$ can be constructed by the sequential application of $\mathscr{A}(\tau)$ and $\mathscr{A}^{*}(\tau)$, i.e. starting from initial condition $\mathbf{u}^{\prime}(0)$, integrate/time step forwards over time interval $\tau$ with the forward system to give $\mathbf{u}^{\prime}(\tau)$, then use this as an initial condition to integrate backwards in time over the same interval using the adjoint system. Application of the joint operator requires only a straightforward extension of the original methodology [1], which was based on the first half of this iteration.

\section{IMPLEMENTATION DETAILS}

Starting from a computer code that solves the unsteady incompressible Navier-Stokes equations, two main modifications are required to produce a code that can perform direct linear stability or transient growth analyses. The first is inner-level modification of the code so that it can integrate the linearized or adjoint linearized equations rather than the nonlinear Navier-Stokes equations. The second aspect is an outer-level algorithm to perform eigenvalue and related computations by repeated calls to the modified Navier-Stokes code. This section of the paper describes practical details of both these modifications.

\subsection{Inner-level code modifications}

3.1.1. Advection terms. We assume that the investigator has an existing Navier-Stokes algorithm where the nonlinear terms can be modified. Codes in which the nonlinear terms are directly evaluated are the most straightforward to adapt. We have used an algorithm with an explicit time integration of the nonlinear terms and where the Stokes operator is inverted using a velocity correction technique $[17,18]$. In our implementation, we adapt the 'convective' form $(\mathbf{u} \cdot \nabla) \mathbf{u}$ of the nonlinearity. Other variants of the nonlinear terms, such as 'rotational' or 'skew-symmetric,' could potentially be used as a starting point; however, we note that adapting these formulations of the nonlinear terms can potentially introduce numerical instabilities, as has been reported by Wilhelm and Kleiser [19].

At the inner-most level, one only needs to replace the code that evaluates the nonlinear term given $\mathbf{u}$ (most likely this is an isolated subroutine), with a code that evaluates $\mathrm{DN}$ or $\mathrm{DN}^{*}$ given $\mathbf{u}$. Employing index notation and the summation convention, the linearized terms in (12) and (15) expressed in the Cartesian coordinates are

$$
\begin{gathered}
\left.(\mathrm{DNu})\right|_{j}=U_{i} \partial_{i} u_{j}+\left(\partial_{i} U_{j}\right) u_{i} \\
\left.\left(\mathrm{DN}^{*} \mathbf{u}\right)\right|_{j}=-U_{i} \partial_{i} u_{j}+\left(\partial_{j} U_{i}\right) u_{i}
\end{gathered}
$$

The components $U_{i}$ are those of a steady or periodic base flow. In Equation (27), we have dropped the primes and stars to emphasize that within this level of the code, separate variables are not used for the perturbation and adjoint fields. If the code evaluates (27a) within each time step, then it advances the forward system, whereas if it evaluates (27b), it advances the adjoint system.

3.1.2. Time integration considerations. It is worth highlighting the implications of the negative sign in front of the time derivative in (18) and the related fact that the initial conditions on the 


\section{DIRECT OPTIMAL GROWTH}

adjoint system (24) are specified at time $\tau$. We envision that a time-stepping code is modified to evaluate the adjoint terms (27b), rather than nonlinear terms or (27a), but keeps the same timestepping algorithm for the viscous and pressure terms. In particular, the code will still march its internal time variable $\bar{t}$ forward from 0 to $\tau$ and effectively solve Equations (18) except with a plus sign in front of the time derivative. This means that when solving the adjoint problem, the internal time variable $\bar{t}$ corresponds to $\tau-t$, and not to $t$. (Writing (18) in terms of $\bar{t}$ restores the plus sign on the time derivative $-\partial u / \partial t \rightarrow \partial u / \partial \bar{t}$ and gives limits on time integration $\bar{t}=0$ to $\bar{t}=\tau$.) Therefore, a time-stepping code automatically evolves fields backwards in time simply by evaluating $\mathrm{DN}^{*}$ in (27b) and otherwise keeping the numerical algorithm the same. When time stepping the adjoint problem, the numerical initial condition, at $\bar{t}=0$, necessarily corresponds to time $t=\tau$. The fact that the internal time variable is $\tau-t$ and not $t$ for the adjoint problem is important for time-dependent base flows.

Typically, the base flow has been previously computed and saved, either by integrating (1) to an attractor or by an iterative method such as Newton's method. If the base flow is steady, the components $U_{i}$ are read, and optionally the derivatives $\partial_{i} U_{j}$ are evaluated and stored for repeated use in the computation of terms (27). If the base flow is time-periodic $U_{i}=U_{i}(t)$ with $U_{i}(t+T)=U_{i}(t)$, then additionally the time variation must be taken into account. This is most easily done by storing the base flow as $M$ equally spaced time slices over one period: $U_{i}^{m}=U_{i}(t=$ $m T / M), m=0, \ldots, M-1$. The base flow may then be reconstructed at each time step by the Fourier interpolation, e.g. $U_{j}(t)=\sum_{m=0}^{M-1} a_{m}(2 \pi t / T) \hat{U}_{j}^{m}$, where $a_{m}$ are the standard trigonometric expressions. For the forward system, $t$ is the internal code time $\bar{t}$, whereas for the adjoint system $t=$ $\tau-\bar{t}$. The derivatives $\partial_{i} U_{j}$ might optionally be computed and stored for the Fourier reconstruction, but this may be relatively expensive when $M$ is large and therefore it can be cheaper to compute the base flow, then differentiate. More generally, and particularly where the base flow may not be time periodic, the base flow at each time step may be computed concurrently from the Navier-Stokes equations.

For transient growth analysis in an unsteady flow, an additional variable must be considered, which is the time offset $t_{0}$ at which the perturbation is initialized relative to the phase of the base flow. In practice, this simply requires time-shifting the base flow by interval $t_{0}$, as will be illustrated in Section 4.3.

3.1.3. Boundary conditions. In almost all cases, some inhomogeneous boundary conditions will be used when solving the nonlinear Navier-Stokes equations for the base flow, since such boundary conditions provide the driving. These boundary conditions must be modified to appropriate homogeneous boundary conditions when solving linear stability and optimal growth problems. In our implementation, and presumably many others, changing boundary conditions from inhomogeneous to homogeneous does not require any code modifications, but rather requires only changes in the problem specification.

Nevertheless, boundary conditions deserve close consideration because these relate to an important issue particular to the optimal growth problem in an open-flow problem with outflow boundary conditions. There are actually two related issues: boundary conditions and domain size. In a standard linear stability analysis where one is only interested in eigenvalues of the forward system, one typically determines appropriate boundary conditions on the perturbation equations by requiring that the total fields, $\mathbf{u}=\mathbf{U}+\varepsilon \mathbf{u}^{\prime}$ and $p=P+\varepsilon p^{\prime}$, satisfy the full boundary conditions on the original problem. This would normally lead to homogeneous Dirichlet boundary conditions for the 


\section{BARKLEY, H. M. BLACKBURN AND S. J. SHERWIN}

perturbation velocity on all boundaries except outflow boundaries where homogeneous Neumanntype boundary condition would arise. It is well established that eigenmodes may have significant amplitude at outflow boundaries and yet the mode structure and corresponding eigenvalue are well converged with respect to domain outflow length when employing Neumann-type boundary conditions, e.g. [20]. We highly recommend such boundary conditions for finding eigenvalues of the forward operator.

However, in the case for optimal growth computations, it is not appropriate for velocity perturbations to have significant amplitude at the outflow boundary. If a perturbation reaches the outflow boundary with non-negligible amplitude, then it is thereafter washed out of the computational domain and the corresponding perturbation energy is lost to the computation. As a result, in optimal growth problems domains must be of sufficient size that velocity perturbation fields reach the outflow boundary with negligible amplitude. In practice, the computational domain for an optimal growth computation must have considerably longer inflow and outflow lengths than for a standard stability computation.

With regard to boundary conditions themselves, motivated by the formulas in Section 2.3, we impose homogeneous Dirichlet boundary conditions on all spatial boundaries, for both the forward and adjoint systems, whether or not Dirichlet boundary conditions are imposed on the corresponding boundaries for the nonlinear Navier-Stokes equations. This approach is both simple and accurate. It avoids the potential difficulties associated with employing the mixed boundary condition that arises as the adjoint boundary conditions to a Neumann outflow condition (see Appendix A).

\subsection{Outer-level considerations}

3.2.1. Preliminary. All outer level algorithms require as an inner operation the numerical approximations to the operators $\mathscr{A}(t)$ and $\mathscr{A}^{*}(t)$. In practice, these consist of one, or possibly two, subroutines that accept a discretized input field $\mathbf{u}$ and return the time-evolved field using the innerlevel modifications discussed in Section 3.1. We will not use separate notation here for discretized fields, thought of as a vectors of length $N$, and continuous fields. We will, however, denote the numerical operators by $\mathbf{A}$ and $\mathbf{A}^{*}$ and their actions on $\mathbf{u}$ in matrix-vector form: $\mathbf{A u}$ and $\mathbf{A}^{*} \mathbf{u}$. We also suppress the time interval over which the operators act. Operator $\mathscr{A}^{*}(t) \mathscr{A}(t)$ is thus expressed as $\mathbf{A}^{*} \mathbf{A u}$ and means compute $\mathbf{A u}$ and then compute $\mathbf{A}^{*}$ on the result.

3.2.2. Eigenvalue evaluation. In this section, we describe a method for finding dominant eigenvalues of $\mathbf{A}$, or $\mathbf{A}^{*} \mathbf{A}$, or even $\mathbf{A}^{*}$. Throughout we let $\mathbf{M}$ be one of these operators. The essence of the method is an standard orthogonal projection of the matrix $\mathbf{M}$ onto a low-dimensional Krylov subspace where direct diagonalization is possible. We first derive algebraic formulas for the projection and then present an algorithm based on the resulting linear algebra. Refer to [21] for a more complete discussion.

Consider the Krylov sequence $\left[\mathbf{u}_{0}, \mathbf{M} \mathbf{u}_{0}, \mathbf{M}^{2} \mathbf{u}_{0}, \ldots, \mathbf{M}^{k} \mathbf{u}_{0}\right]$ starting from some initial vector $\mathbf{u}_{0}$, which we assume to be of unit norm: $\left\|\mathbf{u}_{0}\right\|=1$. This is a collection of $k+1$ vectors obtained from $k$ repeated actions of the operator $\mathbf{M}$. We work with the corresponding sequence of normalized vectors:

$$
\mathbf{T}_{k+1}=\left[\mathbf{u}_{0}, \mathbf{u}_{1}, \mathbf{u}_{2}, \ldots, \mathbf{u}_{k}\right]=\left[\frac{\mathbf{u}_{0}}{\alpha_{0}}, \frac{\mathbf{M} \mathbf{u}_{0}}{\alpha_{1}}, \frac{\mathbf{M} \mathbf{u}_{1}}{\alpha_{2}}, \ldots, \frac{\mathbf{M} \mathbf{u}_{k-1}}{\alpha_{k}}\right]
$$


where the $\alpha_{j}$ are defined so that $\left\|\mathbf{u}_{j}\right\|=1$ for all $j$, and $\alpha_{0}=1$ since we have set $\left\|\mathbf{u}_{0}\right\|=1$. Algebraically, $\mathbf{T}_{k+1}$ is a $N \times(k+1)$ matrix, where the column dimension $N$ is the (large) number of values in the discretized field. The first $k$ of these vectors, $\mathbf{T}_{k}=\left[\mathbf{u}_{0}, \mathbf{u}_{1}, \mathbf{u}_{2}, \ldots, \mathbf{u}_{k-1}\right]$, span the Krylov subspace onto which we project $\mathbf{M}$. The action of $\mathbf{M}$ on $\mathbf{T}_{k}$ is

$$
\mathbf{M T}_{k}=\mathbf{T}_{k+1} \mathbf{D}_{k}^{(k+1)}
$$

where $\mathbf{D}_{k}^{(k+1)}$ is the $(k+1) \times k$ matrix whose elements $D_{i j}=\alpha_{i} \delta_{i, j+1}\left(\delta_{i, j}\right.$ is the Kronecker delta). It generates a scaled left-shift of $k+1$ vectors to $k$ vectors. Equation (28) is the fundamental relationship of a Krylov sequence. All that remains is algebraic manipulation of this equation.

First, we express $\mathbf{T}_{k}$ and $\mathbf{T}_{k+1}$ in terms of their respective (reduced) QR decompositions:

$$
\mathbf{M} \mathbf{Q}_{k} \mathbf{R}_{k}=\mathbf{Q}_{k+1} \mathbf{R}_{k+1} \mathbf{D}_{k}^{(k+1)}
$$

where $\mathbf{Q}_{k}=\left[\mathbf{q}_{0}, \mathbf{q}_{1}, \mathbf{q}_{2}, \ldots, \mathbf{q}_{k-1}\right]$ is $N \times k$ with orthogonal columns, $\mathbf{R}_{k}$ is $k \times k$ upper triangular, and similarly for $\mathbf{Q}_{k+1}$ and $\mathbf{R}_{k+1}$. The $k$ and $(k+1)$ matrices agree where they overlap, e.g. $\mathbf{Q}_{k}$ and $\mathbf{Q}_{k+1}$ are the same except that $\mathbf{Q}_{k+1}$ contains one more column $\mathbf{q}_{k}$.

Defining the $(k+1) \times k$ matrix

$$
\mathbf{H}_{k}^{(k+1)} \equiv \mathbf{R}_{k+1} \mathbf{D}_{k}^{(k+1)} \mathbf{R}_{k}^{-1}
$$

we are able to re-express (29) as

$$
\mathbf{M} \mathbf{Q}_{k}=\mathbf{Q}_{k+1} \mathbf{H}_{k}^{(k+1)}
$$

We will give a simple relation for obtaining the elements of $\mathbf{H}_{k}^{(k+1)}$ momentarily. For now it is sufficient to note that $\mathbf{H}_{k}^{(k+1)}$ is upper Hessenberg and the last row has only one non-zero element that we denote as $h^{*}$, i.e. $h^{*} \equiv h_{k, k-1}$. By letting $\mathbf{H}_{k}$ be the $k \times k$ matrix consisting of all but the last row of $\mathbf{H}_{k}^{(k+1)}$ we can explicitly separate off $\mathbf{q}_{k}$, the last column of $\mathbf{Q}_{k+1}$, from (31) to obtain

$$
\mathbf{M} \mathbf{Q}_{k}=\mathbf{Q}_{k} \mathbf{H}_{k}+h^{*} \mathbf{q}_{k} \hat{\mathbf{e}}_{k}^{\mathrm{T}}
$$

where $\hat{\mathbf{e}}_{k}^{\mathrm{T}}=[0,0, \ldots, 1]$ is a $1 \times k$ unit vector. Equation (32) is the desired projection of $\mathbf{M}$ onto the orthonormal basis $\mathbf{Q}_{k}$. The extent to which the resulting small $k \times k$ matrix $\mathbf{H}_{k}$ fails to exactly capture the action of $\mathbf{M}$ is quantified by the last term.

Let $\mathbf{Y}_{k}=\left[\mathbf{v}_{0}, \mathbf{v}_{1}, \mathbf{v}_{2}, \ldots, \mathbf{v}_{k-1}\right]$ be the $k \times k$ matrix whose columns are normalized eigenvectors of $\mathbf{H}_{k}$, with corresponding eigenvalues $\left(\lambda_{0}, \lambda_{1}, \ldots, \lambda_{k-1}\right)$, then diagonalizing $\mathbf{H}_{k}$ in (32) gives

$$
\begin{aligned}
\mathbf{M} \mathbf{Q}_{k} \mathbf{\Upsilon}_{k} & =\mathbf{Q}_{k} \mathbf{\Upsilon}_{k} \mathbf{\Upsilon}_{k}^{-1} \mathbf{H}_{k} \mathbf{\Upsilon}_{k}+h^{*} \mathbf{q}_{k} \hat{\mathbf{e}}_{k}^{\mathrm{T}} \mathbf{\Upsilon}_{k} \\
\mathbf{M} \Psi_{k} & =\boldsymbol{\Psi}_{k} \boldsymbol{\Lambda}_{k}+h^{*} \mathbf{q}_{k} \hat{\mathbf{e}}_{k}^{\mathrm{T}} \mathbf{\Upsilon}_{k}
\end{aligned}
$$

where $\boldsymbol{\Lambda}_{k}$ is the $k \times k$ block-diagonal eigenvalue matrix of $\mathbf{H}_{k}$ and $\mathbf{Q}_{k} \mathbf{Y}_{k}=\boldsymbol{\Psi}_{k}=\left[\psi_{0}, \psi_{1}\right.$, $\left.\psi_{2}, \ldots, \psi_{k-1}\right]$ is the $N \times k$ matrix of normalized approximate (Ritz) eigenvectors of $\mathbf{M}$. The residual error of eigenpair $\psi_{j}, \lambda_{j}$ is exactly expressible using the last term in (33):

$$
\varepsilon_{j} \equiv\left\|\mathbf{M} \psi_{j}-\lambda_{j} \psi_{j}\right\|=\left\|h^{*} \mathbf{q}_{k} \hat{\mathbf{e}}_{k}^{\mathrm{T}} \mathbf{v}_{j}\right\|=\left|h^{*} \| \mathbf{v}_{j}[k-1]\right|
$$

where the scalar $\mathbf{v}_{j}[k-1]=\hat{\mathbf{e}}_{k}^{\mathrm{T}} \mathbf{v}_{j}$ is the last component of eigenvector $\mathbf{v}_{j}$. 
We assume that complex eigenvectors $\boldsymbol{v}=\boldsymbol{v}^{r} \pm \mathbf{i} \boldsymbol{v}^{i}$ are represented in LAPACK [22] format with real and imaginary parts contained in adjacent vectors: $\boldsymbol{v}^{r}=\mathbf{v}_{j}$ and $\boldsymbol{v}^{i}=\mathbf{v}_{j+1}$. Hence, a normalized complex eigenvector means $\left\|\mathbf{v}_{j}\right\|^{2}+\left\|\mathbf{v}_{j+1}\right\|^{2}=1$ and $|\boldsymbol{v}[k-1]|^{2}=\left|\mathbf{v}_{j}[k-1]\right|^{2}+\left|\mathbf{v}_{j+1}[k-1]\right|^{2}$. Similarly, complex Ritz eigenvectors $\psi$ are represented as pairs $\psi_{j}$ and $\psi_{j+1}$.

Finally, from (30) we obtain an expression for computing the elements $h_{i, j}$ of $\mathbf{H}_{k}^{(k+1)}$ from the elements $r_{i, j}$ of $\mathbf{R}_{k+1}$. Equation (30) can be expressed as $\mathbf{H}_{k}^{(k+1)} \mathbf{R}_{k}=\mathbf{R}_{k+1} \mathbf{D}_{k}^{(k+1)}$. In terms of matrix elements we have, for $0 \leqslant i \leqslant k, 0 \leqslant j \leqslant k-1$,

$$
\sum_{l=0}^{j} h_{i, l} r_{l, j}=\sum_{l=0}^{j} r_{i, l} D_{l, j}=\sum_{l=0}^{j} r_{i, l} \alpha_{l} \delta_{l, j+1}=\alpha_{j+1} r_{i, j+1}
$$

from which one can solve for $h_{i, j}$ :

$$
h_{i, j}=\frac{1}{r_{j, j}}\left(\alpha_{j+1} r_{i, j+1}-\sum_{l=0}^{j-1} h_{i, l} r_{l, j}\right)
$$

3.2.3. The SVD. Consider the previous method applied specifically to the symmetric matrix $\mathbf{M}=\mathbf{A}^{*} \mathbf{A}$. In this case, the eigenvectors $\psi_{j}$ are the right singular vectors $\mathbf{v}_{j}$ of the SVD defined in (8). The left singular vectors are also obtained as a byproduct of the computation, at the cost of a small amount of additional storage and post-processing. Since the action of $\mathbf{A}^{*} \mathbf{A}$ is obtained by applying successively $\mathbf{A}$ to $\mathbf{u}_{j}$ followed by $\mathbf{A}^{*}$, one can store the intermediate vectors after just the forward operator $\mathbf{A}$. Let $\mathbf{w}_{j}=\mathbf{A} \mathbf{u}_{j}$ for each vector $\mathbf{u}_{j}$ in $\mathbf{T}_{k}$. Then,

$$
\mathbf{A T}_{k}=\mathbf{W}_{k}=\left[\mathbf{w}_{0}, \mathbf{w}_{1}, \ldots, \mathbf{w}_{k-1}\right]
$$

Now, perform the manipulations that take $\mathbf{T}_{k}$ to the eigenvectors of $\mathbf{M}$ :

$$
\begin{gathered}
\mathbf{A} \mathbf{Q}_{k} \mathbf{R}_{k}=\mathbf{W}_{k} \\
\mathbf{A} \mathbf{Q}_{k}=\mathbf{W}_{k} \mathbf{R}_{k}^{-1} \\
\mathbf{A} \mathbf{Q}_{k} \mathbf{Y}_{k}=\mathbf{W}_{k} \mathbf{R}_{k}^{-1} \mathbf{Y}_{k} \\
\mathbf{A} \mathbf{V}_{k}=\mathbf{W}_{k} \mathbf{R}_{k}^{-1} \mathbf{Y}_{k}
\end{gathered}
$$

where in the last step we have expressed the matrix of eigenvectors as $\mathbf{V}_{k}=\left[\mathbf{v}_{0}, \mathbf{v}_{1}, \ldots, \mathbf{v}_{k-1}\right]$ rather than $\boldsymbol{\Psi}_{k}$ since these are the right singular vectors in this case. In order for this last equation to be of the form $\mathbf{A} \mathbf{V}_{k}=\mathbf{U}_{k} \boldsymbol{\Sigma}_{k}$, where $\boldsymbol{\Sigma}_{k}=\operatorname{diag}\left\{\sigma_{0}, \ldots, \sigma_{k-1}\right\}$ is the matrix of singular values, we see that

$$
\mathbf{U}_{k}=\mathbf{W}_{k} \mathbf{R}_{k}^{-1} \mathbf{Y}_{k} \boldsymbol{\Sigma}_{k}^{-1}
$$

This equation holds column by column; therefore, given any eigenvector $\mathbf{v}_{j}$ and eigenvalue $\lambda_{j}$ of $\mathbf{H}_{k}$, the left singular vectors are easily obtained from $\mathbf{u}_{j}=\mathbf{W}_{k} \mathbf{R}_{k}^{-1} \lambda_{j}^{-1 / 2} \mathbf{v}_{j}$.

3.2.4. Eigenvalue algorithm. We now give a simple algorithm to compute the $m$ eigenvectors and corresponding eigenvalues of the largest magnitude. At each iteration a Krylov subspace is updated, 


\section{DIRECT OPTIMAL GROWTH}

orthonormalization is performed, eigenvectors of $\mathbf{H}_{k}$ are computed, and the residual norms $\varepsilon_{j}$ are calculated. If the required number of residual norms is not sufficiently small, then another Krylov vector is added. If the Krylov subspace dimension reaches a specified maximum before convergence, the oldest vector is discarded, thus keeping the Krylov subspace size constant. This is equivalent to restarting the method with the second oldest vector in the sequence. Once the required eigenvalues have converged, the Ritz approximations of the correspondent eigenvectors of $\mathbf{M}$ are computed. In the case $\mathbf{M}=\mathbf{A}^{*} \mathbf{A}$, the additional computations for the left singular vectors are also highlighted.

0. Initialize: Set parameters kmax, the maximum dimension of the Krylov subspace, $m$ the number of desired eigenvalue/eigenvectors, and the residual norm tolerance tol. Provide an initial vector $\mathbf{u}_{0}$ with unit norm. Set $k=0$.

1. Update the Krylov subspace $\mathbf{T}_{k+1}=\left[\mathbf{u}_{0}, \mathbf{u}_{1}, \ldots, \mathbf{u}_{k}\right]$ according to

$$
\begin{aligned}
& \text { if }(k<k \text { max }) \text { then } \\
& \quad k=k+1 \\
& \text { else } \\
& \quad \text { for } i=1, k \\
& \quad \alpha_{i-1}=\alpha_{i}, \mathbf{u}_{i-1}=\mathbf{u}_{i}, \quad S V D: \mathbf{w}_{i-1}=\mathbf{w}_{i} \text { if } i \neq k \\
& \text { endif } \quad \text { SVD: } \mathbf{w}_{k-1}=\mathbf{A} \mathbf{u}_{k-1}
\end{aligned}
$$

2. Without destroying $\mathbf{T}_{k+1}$, perform $\mathrm{QR}$ factorization using a modified Gram-Schimdt procedure:

$$
\begin{gathered}
\mathbf{Q}_{k+1}=\mathbf{T}_{k+1}, \quad \mathbf{R}_{k+1}=\mathbf{0}_{k+1} \\
\text { for } i=0, k \\
r_{i, i}=\left|\mathbf{q}_{i}\right|, \quad \mathbf{q}_{i}=\mathbf{q}_{i} / r_{i, i} \\
\text { for } j=i+1, k \\
r_{i, j}=\mathbf{q}_{i} \cdot \mathbf{q}_{j} \\
\mathbf{q}_{j}=\mathbf{q}_{j}-r_{i, j} \mathbf{q}_{i}
\end{gathered}
$$

where $\mathbf{q}_{i}[j]=\mathbf{Q}_{k+1}[i][j]$, and $r_{i, j}=\mathbf{R}_{k+1}[i][j]$

3. Construct the Hessenberg matrix $\mathbf{H}_{k}[i][j]=h_{i, j}$ and compute $h^{*}$

$$
\begin{aligned}
& \text { for } i=0, k-1 \\
& \quad \text { for } j=0, k-1 \\
& \quad h_{i, j}=\left(\alpha_{j+1} r_{i, j+1}-\sum_{l=0}^{j-1} h_{i, l} r_{l, j}\right) / r_{j, j} \\
& h^{*}=\alpha_{k} r_{k, k} / r_{k-1, k-1}
\end{aligned}
$$

where $r_{i, j}$ are known from step 2 .

4. Compute the $k$-normalized eigenvectors $\mathbf{v}_{i}$ and eigenvalues $\lambda_{i}$ of $\mathbf{H}_{k}$ (e.g. using LAPACK).

5. Calculate the $k$ residual norms $\varepsilon_{i}$ as

$$
\varepsilon_{i}=\left|h^{*}\right|\left|\mathbf{v}_{i}[k-1]\right|, \quad 0 \leqslant i<k
$$

and check for convergence by ordering $\varepsilon_{0} \leqslant \varepsilon_{1} \leqslant \cdots \leqslant \varepsilon_{k-1}$ then 


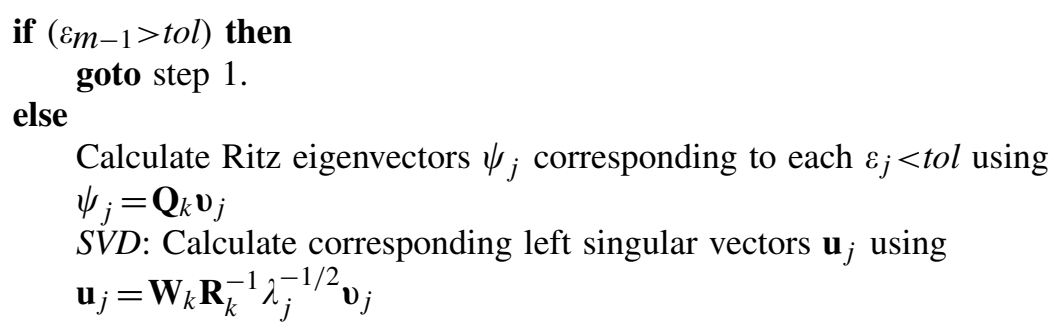

If $\mathbf{M}=\mathbf{A}$, then $\psi_{j}$ and $\lambda_{j}$ in the algorithm correspond to $\tilde{\mathbf{u}}_{j}$ and $\mu_{j}$ in (4). If $\mathbf{M}=\mathbf{A}^{*} \mathbf{A}$, then $\psi_{j}$ and $\lambda_{j}$ correspond to $\mathbf{v}_{j}$ and $\lambda_{j}$ in (6).

3.2.5. Comments. In our experience this algorithm performs very well. Computational cost is dominated by the number of calls to $\mathbf{M}$. The $\mathrm{QR}$ decomposition of $\mathbf{T}_{k+1}$ and the eigenvalue computation of $\mathbf{H}_{k}$ are trivial in comparison. Alternative outer-level methods can be used to find dominant eigenvalues. In particular, the implicitly restarted Arnoldi method, e.g. ARPACK [23], is quite popular. For the types of problems we consider, we find that our method compares favorably with ARPACK in terms of number of calls to $\mathbf{M}$ and it has the advantage that it is easily implemented 'in house' using only basic library calls. It typically requires somewhat more memory than ARPACK.

A question not addressed above is the choice of initial vector $\mathbf{u}_{0}$. We generally use a quasirandom initial vector and rely on the time integration algorithm to enforce solenoidality of the velocity field. If reliable Ritz eigenvectors are required, it is best to ensure that the initial vector is either divergence free or discarded before the Ritz estimate is constructed. Various ways of doing this are (i) analytically define an initial vector that is divergence free; (ii) ensure that the Krylov sequence fills such that the initial vector is discarded before a Ritz estimate is produced; (iii) discard the starting vector and initialize the Krylov sequence after completing one full pass through the time-integration required for the outer loop; and (iv) use a nearby Ritz eigenvector estimate to initialize computations at a new point in parameter space.

\subsection{Discrete inner products and the transpose operations}

The eigenvalue equations for the continuous operator $\mathscr{A}$ do not depend on an inner product. It is not even necessary to have an inner product for the definition of eigenvalues and eigenfunctions. However, for the optimal growth problem, one computes not the eigenvalues of $\mathscr{A}$ but the eigenvalues of $\mathscr{A}^{*} \mathscr{A}$, or equivalently finds the singular values of $\mathscr{A}$. These problems do require an inner product, at least in implied form. Recall from Section 2 that we work with respect to the $L_{2}$ inner product (10) and that the adjoint is defined with respect to this inner product. This fixes the relationship between the discretization of the continuous operators denoted by $\mathbf{A}$ and $\mathbf{A}^{*}$ such that in general $\mathbf{A}^{*} \neq \mathbf{A}^{\mathrm{T}}$. We present formulas for the discrete inner product and discrete transpose.

Here, we will need to distinguish the continuous vectors fields $\mathbf{u}(\mathbf{x})$ from their discrete numerical approximation by $N$-dimensional vectors that we denote by $\mathbf{u}_{N}$. Everything hinges on the fact that if $\mathbf{u}_{N}$ is the discrete approximation of $\mathbf{u}$ and $\mathbf{v}_{N}$ is the discrete approximation of $\mathbf{v}$, then in general the vector dot product $\mathbf{u}_{N}^{\mathrm{T}} \mathbf{v}_{N}$ is not a consistent discrete approximation for the inner product (u, $\left.\mathbf{v}\right)$. Instead the inner product of fields $\mathbf{u}$ and $\mathbf{v}$ takes the discrete form:

$$
\mathbf{u}_{N}^{\mathrm{T}} \mathbf{\Omega} \mathbf{v}_{N} \simeq(\mathbf{u}, \mathbf{v})
$$


where $\boldsymbol{\Omega}$ is a symmetric $N \times N$ matrix, $\boldsymbol{\Omega}^{\mathrm{T}}=\boldsymbol{\Omega}$. If one is formulating the adjoint in an $L_{2}$ inner product, $\boldsymbol{\Omega}$ will be the mass matrix. It need not be diagonal, although it often is.

Following our formulation the matrices $\mathbf{A}$ and $\mathbf{A}^{*}$ are, by definition, consistent approximations to the corresponding operators: $\mathbf{A} \mathbf{u}_{N} \simeq \mathscr{A} \mathbf{u}$ and $\mathbf{A}^{*} \mathbf{v}_{N} \simeq \mathscr{A}^{*} \mathbf{v}$. From this we have

$$
\mathbf{u}_{N}^{\mathrm{T}} \mathbf{\Omega} \mathbf{A}^{*} \mathbf{v}_{N} \simeq\left(\mathbf{u}, \mathscr{A}^{*} \mathbf{v}\right)=(\mathscr{A} \mathbf{u}, \mathbf{v}) \simeq\left(\mathbf{A} \mathbf{u}_{N}\right)^{\mathrm{T}} \boldsymbol{\Omega} \mathbf{v}_{N}=\mathbf{u}_{N}^{\mathrm{T}} \mathbf{A}^{\mathrm{T}} \boldsymbol{\Omega} \mathbf{v}_{N}
$$

Thus, we have the following equations relating matrix elements of $\mathbf{A}^{*}$ to those of $\mathbf{A}^{\mathrm{T}}$ :

$$
\mathbf{\Omega} \mathbf{A}^{*}=\mathbf{A}^{\mathrm{T}} \mathbf{\Omega} \text { or } \quad \mathbf{A}^{*}=\mathbf{\Omega}^{-1} \mathbf{A}^{\mathrm{T}} \mathbf{\Omega}
$$

The eigenvalue equation for $\mathbf{A}^{*} \mathbf{A}$ becomes

$$
\mathbf{\Omega}^{-1} \mathbf{A}^{\mathrm{T}} \mathbf{\Omega A V}=\mathbf{V} \mathbf{\Lambda}
$$

whereas the SVD equation can be expressed as

$$
\mathbf{A V}=\mathbf{U} \boldsymbol{\Sigma} \text { equivalently } \mathbf{U}^{\mathrm{T}} \boldsymbol{\Omega}^{\mathrm{T}} \mathbf{A} \mathbf{V}=\boldsymbol{\Sigma} \text { or } \quad \mathbf{A}=\mathbf{U} \boldsymbol{\Sigma} \mathbf{V}^{\mathrm{T}} \boldsymbol{\Omega}
$$

since $\mathbf{U}$ and $\mathbf{V}$ are orthogonal with respect to $\boldsymbol{\Omega}$. In summary, when one needs to move discrete singular vectors from one side of an equality to the other, matrix $\boldsymbol{\Omega}$ is also required, since it is used in the discrete inner product that defines the discrete adjoint. No special provision is required within the present algorithm, which assumes a continuous-variable adjoint and where the inner product is implicit. However, in a 'discretize-then-transpose' (sometimes referred to as a 'discrete adjoint') approach to computing the SVD based on obtaining $\mathbf{A}$ and $\mathbf{A}^{\mathrm{T}}$ separately (i.e. (38)), $\boldsymbol{\Omega}$ must be explicitly represented.

\section{EXAMPLES AND PRACTICAL GUIDANCE}

In this section, we offer guidance on practical issues and briefly show two examples of optimal perturbation computations. We stress that, as in any computational analysis, one should a priori assume that the results may be in error, e.g. as a result of under-resolution, and be prepared to check, and where possible, cross-check outcomes. For example, it is good practice to re-use 'converged' Ritz eigenvector estimates as restart conditions for the eigensystem analysis and compare the results. We have also found the cross-check described in Section 4.4 to be useful in deciding if spatio-temporal resolution is adequate. In our examples, we have typically applied a residual tolerance $\varepsilon_{i} \leqslant 10^{-6}$. For cases where there is a strong transient growth, we usually observe that the

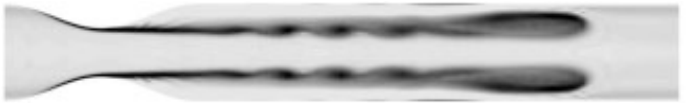

(a)

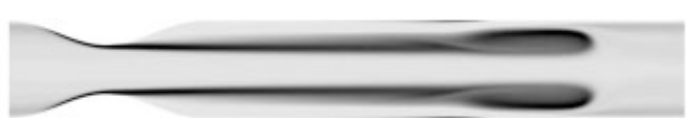

(b)

Figure 3. In computation of an unsteady/periodic base flow, under-resolution may unintentionally excite localized convective instability. These panels show detail snapshot views of vorticity contours in the computation of a pulsatile stenotic flow on the same underlying spectral element mesh, and at the same temporal phase. The element tensor-product basis functions are of order (a) $N=4$ and (b) $N=8$. 
leading eigenvalue converges within approximately 10 iterations. However, for weaker growths, iteration counts can increase to approximately 30 . We have found it best to use a comparatively low Krylov dimension, typically $k=4$. Our experience with a code that is second-order accurate in time [24] and where the time step is Courant-Friedrichs-Lewy-limited has suggested that it is typically appropriate to use a similar time step size when computing base flows, forward, and adjoint linear systems.

\subsection{Base flows}

Base flows are often computed using an unsteady Navier-Stokes solver, especially in the present situation where this forms the basis of a stability analysis code. When computing steady base flows by this method, an obvious requirement is that the flows are very close to their attractor prior to use in stability analysis, and this is easily verified. If the base flow is periodic, however, the simple expedient of allowing a number of integral timescales to elapse (so that transients 'wash out' of the domain) may be inadequate, as under-resolution effects can excite transients that grow and die within each period. Figure 3 illustrates this possibility for a pulsatile axisymmetric base flow [8] in a stenotic/constricted pipe that contains both a vortex ring and an extended shear layer. The passage of each pulse front through the mesh is able to excite Kelvin-Helmholtz-like instability in the shear layer, but this effect disappears as the mesh is refined. Obviously, the flow in Figure 3(a) is an unsuitable basis for a study of convective instability. (Less obviously perhaps, the convective shear-layer oscillation can also affect the asymptotic instability of the flow, which is associated with the vortex ring rather than the shear layer [6].)

\subsection{Backward-facing step}

Our first example of transient growth analysis concerns steady flow over a backward-facing step [10], which is almost infamous as having localized convective instability. For geometries with 1:2 expansion ratio, the first asymptotic instability occurs at $R e=748$, where the two-dimensional flow bifurcates to a steady three-dimensional state [2]. The spectral element mesh for our transient growth study was shown in Figure 1(b). Resolution is concentrated where needed, around the step
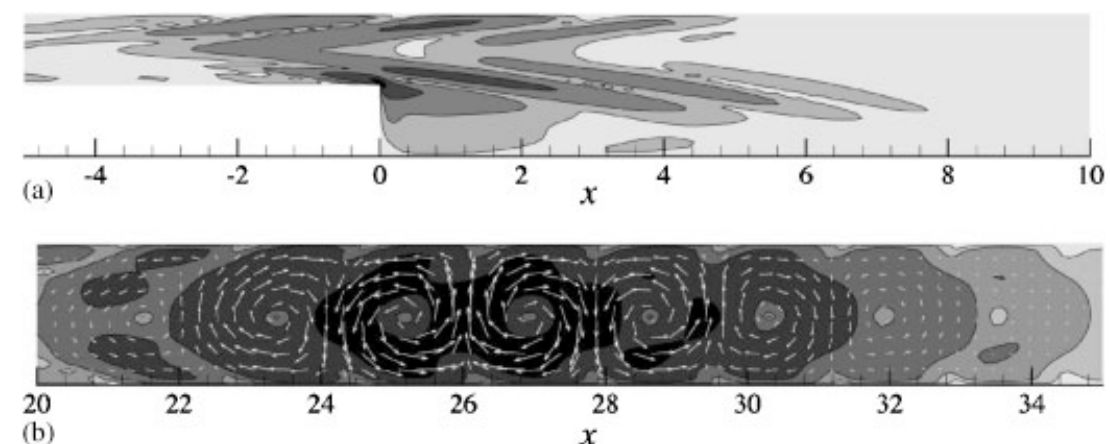

Figure 4. Optimal growth in two-dimensional flow over a backward-facing step at $R e=500$. Decade contours of the logarithm of energy: (a) the global optimal disturbance initial condition (the right singular vector of $\mathscr{A}(\tau)$ ) and (b) the global optimal disturbance outcome (the left singular vector) for $\tau=58.0$, with velocity field vectors overlaid. 


\section{DIRECT OPTIMAL GROWTH}

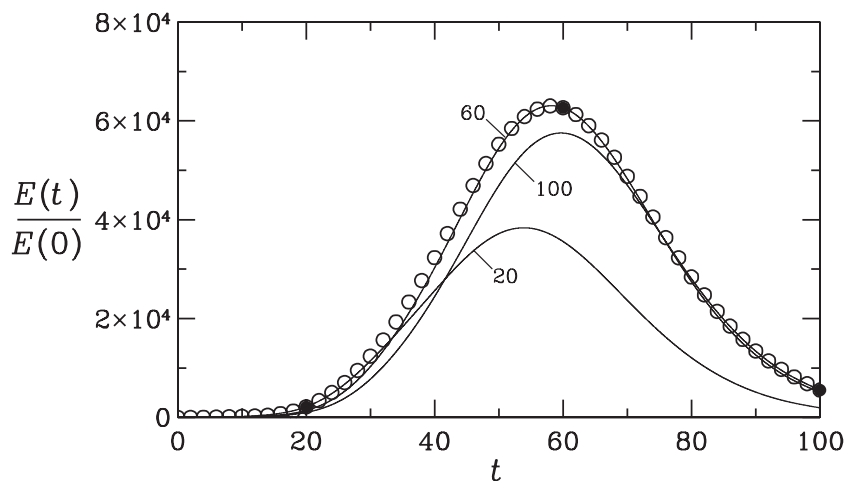

Figure 5. The envelope of two-dimensional optima (circles) for the backward-facing step flow at $R e=500$ together with curves of linear energy evolution starting from three optimal initial conditions for specific values of $\tau: 20,60$, and 100. Solid circles mark the points at which the curves of linear growth osculate the envelope. The global two-dimensional optimum occurs when $\tau=58.0$.

edge, and also the domain is significantly extended in both the up- and downstream directions, when compared with what is required for asymptotic stability analysis of the same flow. This is necessary because the spatial features of the optimal disturbance differ significantly from those of the asymptotic instability, and because we everywhere impose homogeneous Dirichlet boundary conditions on the perturbation field (see Section 3.1.3).

Figure 4 shows contours of energy in the two-dimensional global optimal disturbance initial condition for $R e=500$ and also of the state it evolves to. These correspond, respectively, to the right and left singular vectors of $\mathscr{A}(\tau)$ or, equivalently, to the leading eigenmodes of $\mathscr{A}^{*}(\tau) \mathscr{A}(\tau)$ and $\mathscr{A}(\tau) \mathscr{A}^{*}(\tau)$. Energy in the initial state not only peaks very sharply at the step edge but also extends a number of step-heights upstream and downstream of this point. The initial disturbance is a set of highly stretched rollers that lean backwards against the mean strain-rate field; as time evolves they are tilted upright and gain energy via the inviscid Orr mechanism, evolving to a set of rollers that fill the main channel far downstream, before eventually decaying. See Figure 2 for a sequence of states in this evolution.

Figure 5 illustrates the idea that the leading eigenvalue of $\mathscr{A}^{*}(\tau) \mathscr{A}(\tau)$ gives the envelope of optimal growths as a function of $\tau$. For each $\tau$, the right singular vector of $\mathscr{A}(\tau)$ provides an initial condition that will be evolved by the linearized Navier-Stokes operator to a state that maximizes energy growth when $t=\tau$. At all other times $t \neq \tau$, the energy growth provided by that specific initial condition under the action of the linearized Navier-Stokes operator is below the envelope. In order to obtain the envelope, one must re-solve the eigenproblem with different values of $\tau$. Another feature of Figure 5 is that the energy growths are very large-a sign that in a physical experiment, outcomes will quite likely reflect, or be significantly influenced by, localized convective rather than asymptotic instability.

\subsection{Pulsatile stenotic flow}

When the base flow is $T$-periodic, then an asymptotic stability study is a Floquet analysis, characterized by a constant temporal growth rate and an eigenmode that is also $T$-periodic. In this case, our method delivers the (constant) Floquet multiplier and the eigenmode at a single temporal 


\section{BARKLEY, H. M. BLACKBURN AND S. J. SHERWIN}

phase; to obtain the eigenmode at another phase one must re-do the analysis. However, often the multiplier and a single instance of the eigenmode are sufficient information.

For a transient growth analysis, though, we must not only consider the duration $\tau$ (as is the case for analysis of a steady flow) but also the time $t_{0}$ at which the perturbation is initiated relative to the starting phase of the base flow. As discussed in Section 3.1.2, this time offset is obtained by time-shifting the base flow. We illustrate this with reference to optimal transient growth analysis of a pulsatile axisymmetric stenotic flow [25], as shown in Figure 3, for which we have previously presented the Floquet analysis $[6,8]$. The base flow inlet condition consists of steady (Poiseuille) flow, Reynolds number $R e=\bar{u}_{m} D / v=400$, with a superimposed oscillatory component, relative magnitude 0.75 , i.e.

$$
\bar{u}(t)=\bar{u}_{m}\left\{1+0.75 \sin \left[2 \pi\left(t+t_{0}\right) / T\right]\right\}
$$

where $\bar{u}(t)$ is the cross-sectional area-average flow speed at time $t, \bar{u}_{m}$ is the temporal average of $\bar{u}(t)$ over $T$, and here the dimensionless period $U_{\text {red }}=\bar{u}_{m} T / D=10$. In this case, much as seen in Figure 3(b), the base flow for each pulse generates a leading vortex ring and an extended trailing axisymmetric shear layer. For the combination of parameters chosen here, the critical Reynolds number for asymptotic global instability is $R e_{\mathrm{c}}=590$, and the instability mechanism is associated with alternating tilting of successive vortex rings.

Figure 6 shows the natures of the base flow and optimal disturbance for $\left(t_{0} / T=0.25, \tau / T=\right.$ 0.875 ), close to the global optimum in parameter space. We note that when $t_{0} / T=0.25$ the disturbance is initiated in phase with peak systolic flow, or the highest instantaneous Reynolds

(a)

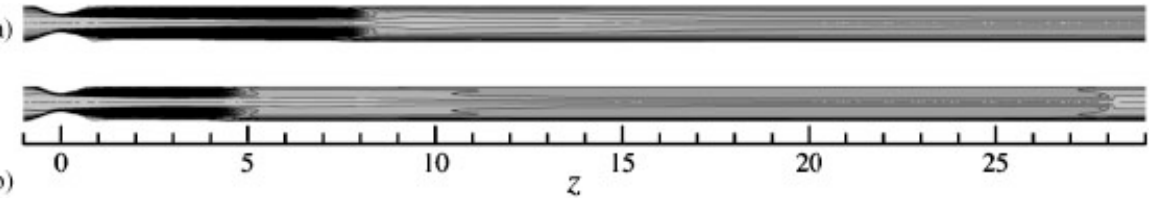

(b)
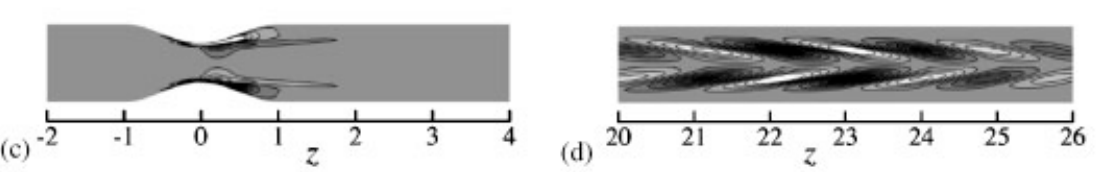

(e)

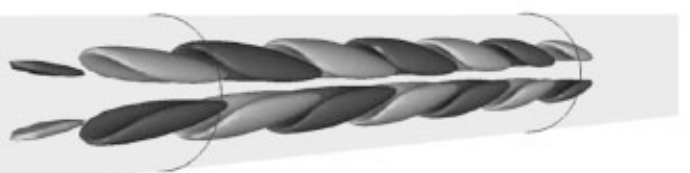

Figure 6. An optimal disturbance to pulsatile stenotic flow, $U_{\text {red }}=10, R e=400$ with initiation at peak systole, $t_{0}=0.25 T$. (a,b), vorticity contours of base flow for (respectively) $t_{0}=0.25 T, t=0$ and $t_{0}=0.25 T, t=0.875 T$. (c), \pm contours of axial velocity in the optimal disturbance initial condition for $\tau=0.875 T$, when the base flow corresponds to (a); and (d), \pm contours of the resulting perturbation at $t=0.875 T$, when the base flow corresponds to (b); axial disturbance wavelength $1.88 D$. (e), perspective view of optimal perturbation at time of maximum growth, shown as \pm isosurfaces of axial velocity, with circumferential lines drawn at $z=20$ and 25 . Note that the contour levels have been chosen independently for each panel to highlight salient features. 


\section{DIRECT OPTIMAL GROWTH}

number. Figure 6(a) shows vorticity contours of the base flow at time $t=0$. At this phase point the pulse front reaches to approximately $z=8$; the leading vortex ring lies in the vicinity $z=7-8$, and it is connected back to the flow separation in the throat by a strong shear layer. In Figure 6(c) one may see positive/negative contours of axial velocity in the optimal perturbation condition (right singular vector) as initiated at the phase point corresponding to Figure 6(a). Figure 6(b) shows vorticity contours in the base flow at the later time $t=\tau=0.875 T$. By this phase, the original pulse front has progressed to approximately $z=28$, and a new pulse front can be seen at approximately $z=4.5$. Figure 6(d) shows positive/negative contours of axial velocity in the optimal disturbance outcome (left singular vector) at $t=\tau=0.875 T$, i.e. corresponding to the base flow phase of Figure $6(\mathrm{~b})$, and it is evident that the perturbation resides in the shear-layer upstream of the leading pulse front (or vortex ring). A perspective view of this disturbance is provided in Figure 6(e). The optimal perturbation produces a sinuous disturbance to an originally axisymmetric shear layer, which first grows in energy as it advects downstream from near the flow separation line, peaks, then dissipates as it advects further downstream.

Now the optimal growth is a function of both $\tau$ and $t_{0}$; therefore, there is a separate envelope $G(\tau)$ for each $t_{0}$, as illustrated in Figure 7(a). Combining results for different $\tau$ and $t_{0}$, we obtain a contour plot of $G\left(\tau, t_{0}\right)$, as shown in Figure $7(\mathrm{~b})$, from which it is apparent that the global optimum $G_{\max }$ occurs for $\tau \approx 0.83 T, t_{0} \approx 0.25 T$, and also that this energy growth again is very large, $G_{\max } \sim 10^{10}$ [25]. Such exceedingly large sensitivity to perturbation suggests that the concept of a critical parameter could be somewhat irrelevant in practice.

\subsection{Checking energy growth and singular vectors}

After bootstrapping a new transient growth analysis code so that it reproduces standard results in simple geometries (e.g. [26]), one faces the question: how might I check the prediction in a novel situation? Fortunately, a simple cross-check exists. Using the leading eigenvector of $\mathscr{A}^{*}(\tau) \mathscr{A}(\tau)$
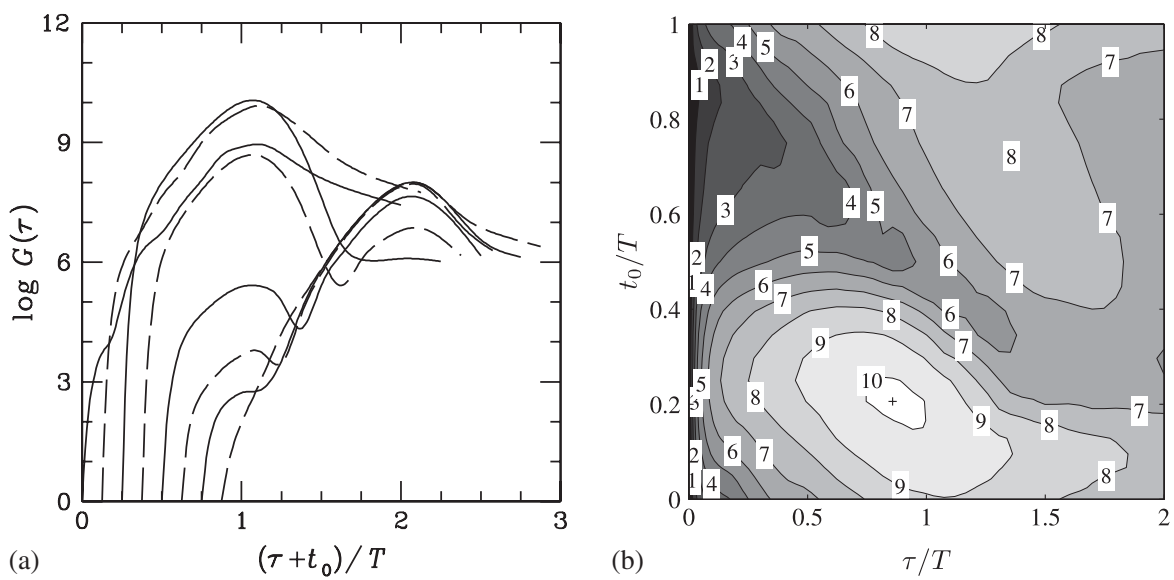

Figure 7. Optimal growth envelopes for single-harmonic pulsatile stenotic flow. When the base flow is periodic (more generally, time varying), an additional parameter $t_{0}$ enters the analysis, where $t_{0}$ is the phase at which the perturbation is introduced, relative to the starting time of the base flow. (a) Shows growth envelopes for eight different initial phases, while (b) gives contours of $\log G$ as a function of growth interval and starting phase. 
as initial condition for evolution under the linearized Navier-Stokes equations (i.e. $\mathscr{A}(\tau)$ alone), one computes the ratio of initial and final kinetic energies using the discrete equivalent of the energy norm assumed in the analysis. Starting from a continuous-variable statement, this is

$$
\frac{E(\tau)}{E(0)}=\frac{(\mathbf{u}(\tau), \mathbf{u}(\tau))}{(\mathbf{u}(0), \mathbf{u}(0))}=\frac{(\mathscr{A}(\tau) \mathbf{u}(0), \mathscr{A}(\tau) \mathbf{u}(0))}{(\mathbf{u}(0), \mathbf{u}(0))} \simeq \frac{\mathbf{u}_{N}^{\mathrm{T}}(\tau) \Omega \mathbf{u}_{N}(\tau)}{\mathbf{u}_{N}^{\mathrm{T}}(0) \Omega \mathbf{u}_{N}(0)}
$$

Assuming that $\mathbf{u}(0)$ was a right singular vector $\mathbf{v}_{N}$ and using (36)-(39) this should be

$$
\frac{E(\tau)}{E(0)} \simeq \frac{\left(\mathbf{U} \boldsymbol{\Sigma} \mathbf{V}^{\mathrm{T}} \boldsymbol{\Omega} \mathbf{v}_{N}\right)^{\mathrm{T}} \boldsymbol{\Omega} \mathbf{U} \boldsymbol{\Sigma} \mathbf{V}^{\mathrm{T}} \boldsymbol{\Omega} \mathbf{v}_{N}}{\mathbf{v}_{N}^{\mathrm{T}} \boldsymbol{\Omega} \mathbf{v}_{N}}=\mathbf{v}_{N}^{\mathrm{T}} \boldsymbol{\Omega} \mathbf{V} \boldsymbol{\Sigma} \mathbf{U}^{\mathrm{T}} \boldsymbol{\Omega} \mathbf{U} \boldsymbol{\Sigma} \mathbf{V}^{\mathrm{T}} \boldsymbol{\Omega} \mathbf{v}_{N}=\mathbf{v}_{N}^{\mathrm{T}} \boldsymbol{\Omega} \mathbf{V} \boldsymbol{\Lambda} \mathbf{V}^{\mathrm{T}} \boldsymbol{\Omega} \mathbf{v}_{N}=\lambda_{i}
$$

where $\lambda_{i}$ is the eigenvalue of $\mathbf{A}^{*} \mathbf{A}$ that corresponds to the index of $\mathbf{v}_{N}$ in the columns of $\mathbf{V}$. Thus, one checks that the linear energy growth does in fact match the computed eigenvalue.

\section{CONCLUSIONS}

In this study, we have shown how existing timestepping-based techniques originally developed for large-scale asymptotic stability analysis in general flows may be extended to optimal transient growth analysis. In the process, we have described the derivation of the adjoint problem in primitivevariable form with particular consideration given to boundary conditions, a topic that often does not arise in simpler flows e.g. where spatial periodicity may be assumed. Although asymptotic stability analysis amounts to finding the leading components of the eigenvalue decomposition of the linearized Navier-Stokes operator, optimal transient growth analysis corresponds to finding the leading SVD components of its action over a finite-time interval. Together, the pair of techniques described for determining initial conditions that provide optimal asymptotic and finite-time growth in non-parallel flows constitutes an area of study for which we have coined the term 'direct optimal growth' analysis.

\section{APPENDIX A}

\section{A.1. Outflow boundary conditions}

In principle, one could be interested in a variety of different boundary conditions on the forward problem. For reference we derive adjoint boundary conditions corresponding to homogeneous Neumann outflow boundary conditions on the forward problem. The outflow boundary conditions considered are the traction free boundary condition

$$
\operatorname{Re}^{-1} \partial_{n} \mathbf{u}-p \hat{\mathbf{n}}\left(\partial \Omega_{\mathrm{o}}, t\right)=0
$$

or the more stringent outflow boundary condition (imposed in our implementation)

$$
\partial_{n} \mathbf{u}\left(\partial \Omega_{\mathrm{o}}, t\right)=p\left(\partial \Omega_{\mathrm{o}}, t\right)=0
$$

where $\Omega_{\mathrm{o}}$ is the portion of the domain boundary on which the outflow condition is imposed and $\mathbf{n}$ is the unit outward normal. 
Recall from Section 2.3 that the adjoint boundary conditions are those which necessarily force (20) to vanish. The portion of the integral corresponding to the outflow boundary is

$$
\int_{0}^{\tau} \mathrm{d} t \oint_{\partial \Omega_{0}} \mathrm{~d} S \hat{\mathbf{n}} \cdot\left\{-\mathbf{U}\left(\mathbf{u} \cdot \mathbf{u}^{*}\right)+\mathbf{u} p^{*}-\mathbf{u}^{*} p+R e^{-1}\left((\nabla \mathbf{u})^{\mathrm{T}} \cdot \mathbf{u}^{*}-\left(\nabla \mathbf{u}^{*}\right)^{\mathrm{T}} \cdot \mathbf{u}\right)\right\}
$$

which can be simplified to

$$
\int_{0}^{\tau} \mathrm{d} t \oint_{\partial \Omega_{0}} \mathrm{~d} S\left\{-(\mathbf{U} \cdot \hat{\mathbf{n}})\left(\mathbf{u} \cdot \mathbf{u}^{*}\right)+\left(\mathbf{u} p^{*}-\mathbf{u}^{*} p\right) \cdot \hat{\mathbf{n}}+\operatorname{Re}^{-1}\left(\left(\partial_{n} \mathbf{u}\right) \cdot \mathbf{u}^{*}-\left(\partial_{n} \mathbf{u}^{*}\right) \cdot \mathbf{u}\right)\right\}
$$

Using the forward boundary condition, $\partial_{n} \mathbf{u}=p=0$ or $R e^{-1} \partial_{n} \mathbf{u}-p \hat{\mathbf{n}}=0$, this becomes

$$
\int_{0}^{\tau} \mathrm{d} t \oint_{\partial \Omega_{0}} \mathrm{~d} S\left\{\left(-(\mathbf{U} \cdot \hat{\mathbf{n}}) \mathbf{u}^{*}-\operatorname{Re}^{-1} \partial_{n} \mathbf{u}^{*}\right) \cdot \mathbf{u}+\mathbf{u} p^{*} \cdot \hat{\mathbf{n}}\right\}
$$

In order for this to vanish identically, it is necessary that the traction free adjoint boundary condition

$$
\left((\mathbf{U} \cdot \hat{\mathbf{n}}) \mathbf{u}^{*}+R e^{-1} \partial_{n} \mathbf{u}^{*}-p^{*} \hat{\mathbf{n}}\right)\left(\partial \Omega_{\mathrm{o}}, t\right)=0
$$

is imposed or the more stringent boundary conditions

$$
\left((\mathbf{U} \cdot \hat{\mathbf{n}}) \mathbf{u}^{*}+\operatorname{Re}^{-1} \partial_{n} \mathbf{u}^{*}\right)\left(\partial \Omega_{\mathrm{o}}, t\right)=p^{*}\left(\partial \Omega_{\mathrm{o}}, t\right)=0
$$

are enforced.

These are the homogeneous adjoint boundary conditions corresponding to outflow boundary conditions (A1) and (A2). Only for $\mathbf{U} \cdot \hat{\mathbf{n}}=0$ on the outflow does the adjoint boundary condition become the same as the forward boundary condition.

\section{ACKNOWLEDGEMENTS}

D. B. wishes to acknowledge support from an Senior Fellowship of the City of Paris. H. M. B. wishes to acknowledge U.K. EPSRC grant EP/E006493/1 in financial support of a Visiting Fellowship. S. J. S. would like to acknowledge financial support from an EPSRC Advanced Research Fellowship. We would also like to acknowledge helpful discussions with Bruno Souza Carmo and Dr Ati Sharma.

\section{REFERENCES}

1. Tuckerman LS, Barkley D. Bifurcation analysis for timesteppers. In Numerical Methods for Bifurcation Problems and Large-Scale Dynamical Systems, Doedel E, Tuckerman LS (eds). Springer: Berlin, 2000; 453-566.

2. Barkley D, Gomes MGM, Henderson RD. Three-dimensional instability in flow over a backward-facing step. Journal of Fluid Mechanics 2002; 473:167-190.

3. Barkley D, Henderson RD. Three-dimensional Floquet stability analysis of the wake of a circular cylinder. Journal of Fluid Mechanics 1996; 322:215-241.

4. Blackburn HM. Three-dimensional instability and state selection in an oscillatory axisymmetric swirling flow. Physics of Fluids 2002; 14(11):3983-3996.

5. Blackburn HM, Lopez JM. The onset of three-dimensional standing and modulated travelling waves in a periodically driven cavity flow. Journal of Fluid Mechanics 2003; 497:289-317.

6. Blackburn HM, Sherwin SJ. Instability modes and transition of pulsatile stenotic flow: pulse-period dependence. Journal of Fluid Mechanics 2007; 573:57-88.

7. Elston JR, Blackburn HM, Sheridan J. The primary and secondary instabilities of flow generated by an oscillating circular cylinder. Journal of Fluid Mechanics 2006; 550:359-389. 


\section{BARKLEY, H. M. BLACKBURN AND S. J. SHERWIN}

8. Sherwin SJ, Blackburn HM. Three-dimensional instabilities and transition of steady and pulsatile flows in an axisymmetric stenotic tube. Journal of Fluid Mechanics 2005; 533:297-327.

9. Åkervik E, Hœpffner J, Ehrenstein U, Henningson DS. Optimal growth, model reduction and control in a separated boundary-layer flow using global eigenmodes. Journal of Fluid Mechanics 2007; 579:305-314.

10. Blackburn HM, Barkley D, Sherwin SJ. Convective instability and transient growth in flow over a backward-facing step. Journal of Fluid Mechanics 2008; 603:271-304.

11. Ehrenstein U, Gallaire F. On two-dimensional temporal modes in spatially evolving open flows: the flat-plate boundary layer. Journal of Fluid Mechanics 2005; 536:209-218.

12. Marquet O, Sipp D, Jacquin L. Global optimal perturbations in a separated flow over a backward-rounded-step. Thirty-sixth AIAA Fluid Dynamics Conference and Exhibit, San Francisco, 2006; Paper No. 2006-2879.

13. Karniadakis GE, Sherwin SJ. Spectral/hp Element Methods for Computational Fluid Dynamics (2nd edn). Oxford University Press: Oxford, 2005.

14. Cossu C, Chomaz JM. Global measures of local convective instabilities. Physical Review Letters 1997; 78: 4387-4390.

15. Schmid PJ, Henningson DS. Stability and Transition in Shear Flows. Springer: Berlin, 2001.

16. Trefethen LN, Trefethen AE, Reddy SC, Driscoll TA. Hydrodynamic stability without eigenvalues. Science 1993; 261:578-584.

17. Karniadakis GE, Israeli M, Orszag SA. High-order splitting methods for the incompressible Navier-Stokes equations. Journal of Computational Physics 1991; 97(2):414-443.

18. Guermond JL, Shen J. Velocity-correction projection methods for incompressible flows. SIAM Journal on Numerical Analysis 2003; 41:112-134.

19. Wilhelm D, Kleiser L. Stability analysis for different formulations of the nonlinear term in $P_{N}-P_{N-2}$ spectral element discretizations of the Navier-Stokes equations. Journal of Computational Physics 2001; 174:306-326.

20. Barkley D. Confined three-dimensional stability analysis of the cylinder wake. Physical Review E 2007; 71:017301.

21. Saad Y. Numerical Methods for Large Eigenvalue Problems. Wiley: New York, 1992.

22. Anderson E, Bai Z, Bischof C, Blackford S, Demmel J, Dongarra J, Du Croz J, Greenbaum A, Hammarling S, McKenney A, Sorensen D. LAPACK User's Guide (3rd edn). SIAM: Philadelphia, PA, 1999.

23. Lehoucq RB, Sorenson DC, Yang C. ARPACK Users' Guide. SIAM: Philadelphia, PA, 1998.

24. Kaiktsis L, Karniadakis GE, Orszag SA. Onset of three-dimensionality, equilibria, and early transition in flow over a backward-facing step. Journal of Fluid Mechanics 1991; 231:501-528.

25. Blackburn HM, Sherwin SJ, Barkley D. Convective instability and transient growth in steady and pulsatile stenotic flows. Journal of Fluid Mechanics 2008; 603:271-304.

26. Butler KM, Farrell BF. Three-dimensional optimal perturbations in viscous shear flow. Physics of Fluids A 1992; 4(8):1637-1650. 